\title{
Morphine compared to placebo for procedural pain in preterm infants: safety, efficacy and equipoise
}

\author{
Omri David Soffer ${ }^{1} \cdot$ Laura Cornelissen ${ }^{2} \cdot$ Christy Cummings $^{1} \cdot$ Charles Berde $^{2}$
}

Received: 14 July 2019 / Accepted: 20 July 2019 / Published online: 27 August 2019

(c) The Author(s) 2019. This article is published with open access

Manuscript citation: C. Hartley, F. Moultrie, A. Hoskin, G. Green, V. Monk, J. L. Bell, et al. Analgesic efficacy and safety of morphine in the Procedural Pain in Premature Infants (Poppi) study: randomised placebo-controlled trial. Lancet. 2018;392:2595-2605

\section{Type of investigation}

Treatment.

\section{Question}

Among nonventilated preterm infants, who are less than 32 weeks of gestation or with birth weight less than $1501 \mathrm{~g}$, undergoing an eye examination to detect retinopathy of prematurity (ROP) or heel lancing, will a $0.1 \mathrm{mg} / \mathrm{kg}$ single oral dose of morphine, compared to a placebo solution, be safe, and provide analgesic efficacy demonstrated by Premature Infant Pain Profile-Revised scoring system (PIPP-R) and noxious-evoked brain activity (nEBA) while maintaining physiological stability?

\section{Methods}

Design: Randomized, double-blinded, placebo-controlled clinical trial.

Omri David Soffer

Omri.soffer@childrens.harvard.edu

1 Division of Newborn Medicine, Boston Children's Hospital, Boston, MA, USA

2 Division of Pain Medicine, Department of Anesthesiology, Critical Care and Pain Medicine, Boston Children's Hospital, Boston, MA, USA
Follow-up period: $24 \mathrm{~h}$ after the eye exam and heel lancing.

Setting: Single-center, academically affiliated neonatal intensive care unit (NICU).

Patients: (1) Inclusion criteria: Nonventilated preterm infant born at less than 32 weeks' gestation OR with a birth weight of less than $1501 \mathrm{~g}$ and aged 34-42 weeks' gestation (postmenstrual age) AND requiring eye exam for ROP screening or a heel lance. (2) Exclusion criteria: Infants with grade 3 or 4 intraventricular hemorrhage, short bowel syndrome, nil per os status, congenital/genetic conditions with probable neurodevelopmental manifestations, sedative or opiate exposure 24 and $72 \mathrm{~h}$ prior to ROP examination or heel lancing, respectively, documented opiate sensitivity, history of maternal opiate exposure either in utero or while breastfeeding, mechanical ventilation at the time of the trial.

Intervention: Preterm infants were randomized to a morphine group (received a single oral dose of morphine sulfate $0.1 \mathrm{mg} / \mathrm{kg}$ ) or control group (received oral placebo solution similar in color, odor, and volume). Solutions were given approximately $1 \mathrm{~h}$ prior to ROP exam and heel lancing. Investigators and clinical team were blinded.

\section{Outcomes}

Co-primary outcomes: Premature Infant Pain ProfileRevised (PIPP-R score after ROP exam); magnitude of nEBA after heel lancing.

Secondary outcomes: limb reflex withdrawal, PIPP-R scores after heel lancing, clinical stability determined by episodes of apnea, bradycardia, tachycardia, desaturation and escalation in respiratory support during the 6 and 24 $\mathrm{h}$ after the clinical procedures, drug safety determined by the incidence of apnea requiring noninvasive positive pressure ventilation and hypotension requiring vasopressors $24 \mathrm{~h}$ after administering morphine or placebo.

Analysis and sample size: 132 infants were needed to detect a 2-point reduction in PIPP-R score, defined as a 
Table. 1 Efficacy outcomes compared between the morphine group and the placebo group

\begin{tabular}{lllc}
\hline Efficacy outcomes & $\begin{array}{l}\text { Morphine group } \\
(n=15)\end{array}$ & $\begin{array}{l}\text { Placebo group } \\
(n=15)\end{array}$ & $\begin{array}{l}\text { Mean/median } \\
\text { difference }\end{array}$ \\
\hline PIPP-R score after ROP exam (mean \pm SD) & $11.1 \pm 3.2$ & $10.5 \pm 3.4$ & 0.5 \\
nEBA after heel lancing (median, IQR) & $0.99(0.4-1.56)$ & $0.75(0.33-1.22)$ & 0.25 \\
PIPP-R score after heel lancing (mean \pm SD) & $7.9 \pm 3.4$ & $8.5 \pm 3.9$ & -0.6 \\
$\begin{array}{l}\text { Reflex withdrawal magnitude after heel lancing } \\
\text { (median, IQR) }\end{array}$ & $24.8(19.7-44.8)$ & $12.4(6.1-46.3)$ & 8.9 \\
\hline
\end{tabular}

$P I P P-R$ Premature Infant Pain Profile-Revised scoring system, $R O P$ retinopathy of prematurity, $n E B A$ noxious-evoked brain activity

clinically significant reduction, from a conservative postretinopathy of prematurity screening mean PIPP-R score of 8.3 (SD 3.5) from a previous study, with power of $90 \%$ and alpha of 0.05 (two-tailed). Additionally, a $40 \%$ relative risk reduction in noxious-evoked brain activity was defined as clinically significant with $90 \%$ power and $5 \%$ significance. The investigators aimed for a sample size of 156 infants to account for multiple births and anticipated $10 \%$ loss of follow-up. Values were reported in mean and median values. PIPP-R scores were compared between the two groups using a $t$ test. The magnitudes of noxious-evoked brain activity were compared between the groups using a Wilcoxon rank-sum test.

Patient follow-up: Infants were followed for $24 \mathrm{~h}$ after ROP examination and heel lancing after receiving morphine or placebo oral solutions. The trial was stopped early at $19 \%$ enrollment (30 infants) by the study's Data Monitoring Committee 1 year after enrollment had commenced using predefined stopping criteria due to a concern for patient safety (profound respiratory adverse effects) without evidence of analgesic efficacy.

\section{Main results}

The baseline characteristics and demographics of the study participants were balanced on some aspects (e.g. gestational and postnatal ages, birth weight) and unbalanced in others as expected in a small sample size (15 patients in each group). For example: the number of infants who required ventilator support (6 in the morphine group and 12 in the placebo group) and the number of infants who were previously exposed to morphine (4 in the morphine group and 7 in the placebo group).

The efficacy outcomes showing no difference between morphine and placebo group is shown in Table 1. The safety outcomes, reported as median of the standarized difference in number of episodes before and after intervention, have shown significant higher episodes in desaturation $0.66(95 \%$ CI: $0.36-1.00, \quad p=0.0007)$ and bradycardia 0.33 (CI: $0.00-0.98, p=0.07$ ) in the morphine group at $6 \mathrm{~h}$ as well as $24 \mathrm{~h}$ for desaturation 0.33 (CI: $0.03-0.75, p=0.019$ ) and bradycardia 0.43 (CI: $0.00-1.00$, $p=0.019$ ).

\section{Study conclusion}

Oral morphine administration to nonventilated premature infants is not recommended due to its potential harm and lack of analgesic effect at the proposed dosage $(0.1 \mathrm{mg} / \mathrm{kg})$.

\section{Commentary}

Previous clinical trials have yet to demonstrate effective, minimal risk analgesic management in neonates undergoing ROP eye examination [1]. Accurate pain assessment in infants is challenging for clinicians since infants cannot provide self-report. Indirect measures of pain assessment such as heart rate, oxygen saturation, and body movement are used as surrogate measures [2]. These indirect measures are mediated at the spinal and subcortical level, and may not accurately reflect pain perception at the level of the cortex [3]. To overcome this barrier, the study investigators used an innovative study design incorporating multimodal measures of pain assessment including EEG-based indices in combination with a traditional pain score (PIPP-R). The study hypothesis was that analgesic efficacy, measured through a reduction of cortical nEBA and PIPP-R scores, would be seen in the morphine arm, compared to the placebo arm.

The study showed that reduction in PIPP-R score, noxious-evoked brain activity magnitude and EMG reflex withdrawal magnitude did not differ significantly between the morphine and the placebo group. However, the trial had to be stopped early according to predefined stopping criteria due to clinical instability of patients in the morphine group (profound respiratory adverse effects).

No other trials to date have been conducted to specifically assess the analgesic efficacy and safety of morphine administration for ROP examination. Several randomized 
controlled trials have evaluated the analgesic efficacy of acetaminophen for ROP exam to demonstrate a possible reduction in Premature Infant Pain Profile (PIPP) score during the examination. However, those trials demonstrated either modest reduction in PIPP score after acetaminophen was given compared to sterile water or no significant reduction in PIPP score followed by oral sucrose solution $[4,5]$. Moreover, those trials only utilized the PIPP scoring system to assess analgesic effect, ultimately relying on behavioral and autonomic activity. There is one ongoing study that may provide more informative pain assessment in newborns using different modalities to assess infants' nociceptive and physiologic responses to noxious stimuli [6].

The study findings call into question the safety of morphine, at the dose used, for acute procedural pain relief in nonventilated preterm infants, given the notable increase in adverse effects. An enteral dose of $0.1 \mathrm{mg} / \mathrm{kg}$ of morphine may have been a high initial dose, especially given a significant number of the study participants were morphine naïve (73\%), potentially leading to increased apnea, bradycardia, and desaturation episodes in this group. Therefore, considering the clinical instability of many of the infants receiving enteral morphine, a lower dose may have led to a more preferable safety outcome, although unlikely to have demonstrated analgesic efficacy. The trial was left underpowered, resulting in an inability to make confident conclusions regarding the analgesic efficacy of morphine in this population.

A recent meta-analysis of randomized trials compared topical ophthalmic anesthetics to various pain-relieving interventions for ROP examination. Despite evidence that multisensory pain treatments (i.e. oral dextrose solution, expressed breast milk, rocking, singing, nonnutritive sucking, swaddling, and acetaminophen) may reduce infants' pain scores to varying degrees, no specific treatment necessarily provided clinically meaningful pain relief. The majority of infants in the trials had a mean PIPP score higher than 12 (i.e. moderate to severe pain) [1]. Therefore, safe and effective analgesics for nonventilated preterm infants undergoing ROP exam is still needed.

In conclusion, this trial is notable for its novel use of innovative pain assessment tools (EEG and EMG), in addition to the more subjective PIPP-R score. Ongoing trials will hopefully provide more informative and objective pain assessment in newborns using different modalities to assess nociceptive and physiological activity, when PIPP-R scores or other behavioral-based pain assessments are not applicable such as in a state of heavy sedation or neuromuscular blockade. Brain-based assessment modalities such as EEG may also provide valuable information about pain in infants and guide optimal management.

\section{EBM lesson: Ethical challenges in conducting randomized controlled trials of analgesics in newborns}

Conducting a randomized controlled trial of analgesics compared to placebo involving newborns has distinct ethical challenges. In clinical research, equipoise assumes genuine uncertainty regarding treatment superiority. When equipoise has been disrupted, the trial should then be stopped according to predetermined criteria and interim safety analysis, as in the POPPI Trial. The justification for placebo controls should be determined on the basis of the research question, and current standard of care. However, the lack of a clear standard of care for treatment of neonatal pain raises a valid concern in clinical trials; equipoise is further complicated when data are lacking about either treatment in a randomized clinical trial. The POPPI trial was designed as a standard versus experimental study (i.e. ophthalmic topical anesthetics and swaddling versus morphine administration). Given the lack of standard of care for these clinical situations, one therapeutic measure was compared with another; both are considered "off-label." Without a standard treatment for pain control, then a placebo might be considered ethically acceptable. Using the POPPI trial as an example, if most providers did not use topical ophthalmic anesthetics, then it would be ethically permissible to test morphine against placebo. If a proven effective and safe means of pain control for a particular indication in a particular population exists, then a placebo-controlled trial would be ethically impermissible and new treatment must be tested against the efficacious treatment. Testing a new therapy as potential adjuvant to the "standard" therapy allows all subjects to receive the "standard" therapy, thus an ethically permissible approach. In the POPPI trial, this strategy was utilized where all the study participants were swaddled and received topical ophthalmic anesthetics [7, 8].

The American Academy of Pediatrics (AAP) has outlined conditions under which the use of placebos may be ethically acceptable: (1) lack of accepted therapy for the condition and the agent under study may modify the course of the disease process (in this case, procedural pain); (2) questionable efficacy of the commonly used therapy; (3) known adverse events for the commonly used therapy; (4) the placebo is used to identify undesirable side effects produced by adding a new treatment; and (5) the disease process is characterized by frequent, spontaneous exacerbations and remissions, without satisfactory therapy [9]. Considering procedural pain as the disease process in this case, all the outlined criteria above may be applicable to the POPPI trial. As pain is undeniably unfavorable, potentially effective and safer analgesic methods should be investigated.

In general, the efficacy and safety of an analgesic should be compared against the best current therapeutic methods. 
The use of placebo may be permissible in studies where no proven therapeutic intervention exists; in other words, a true state of equipoise [8]. Why then is a placebo control group acceptable if pain is ethically and morally unacceptable and analgesia is preferable; why not just treat the patient? The answer lies in the uncertainty of the benefits gained and risks involved in the proposed treatment, given the limitations of current assessment methods. Out of necessity, unapproved and understudied drugs are commonly used in neonatal care; going forward clinical research of drugs in newborns will be even more important, as well as input from the institutional board governing these studies [8].

In summary, the POPPI trial demonstrated various ethical challenges in designing clinical trials involving newborns, including justifying placeboes, defining equipoise and highlights the need for future studies in this area.

Acknowledgements The Journal Club is a collaboration between the American Academy of Pediatrics-Section of Neonatal Perinatal Medicine and the International Society for Evidence-Based Neonatology (EBNEO.org).

\section{Compliance with ethical standards}

Conflict of interest The authors declare that they have no conflict of interest.

Publisher's note: Springer Nature remains neutral with regard to jurisdictional claims in published maps and institutional affiliations.

Open Access This article is licensed under a Creative Commons Attribution 4.0 International License, which permits use, sharing, adaptation, distribution and reproduction in any medium or format, as long as you give appropriate credit to the original author(s) and the source, provide a link to the Creative Commons license, and indicate if changes were made. The images or other third party material in this article are included in the article's Creative Commons license, unless indicated otherwise in a credit line to the material. If material is not included in the article's Creative Commons license and your intended use is not permitted by statutory regulation or exceeds the permitted use, you will need to obtain permission directly from the copyright holder. To view a copy of this license, visit http://creativecommons. org/licenses/by/4.0/.

\section{References}

1. Disher T, Cameron C, Mitra S, Cathcart K, Campbell-Yeo M. Painrelieving interventions for retinopathy of prematurity: a metaanalysis. Pediatrics 2018;142:e20180401.

2. Cong X, McGrath J, Cusson R, Zhang D. Pain assessment and measurement in neonates: an updated review. Adv Neonatal Care. 2013;13:379-95.

3. Fitzgerald M. What do we really know about newborn infant pain? Exp Physiol 2015;100:1451-7.

4. Kabataş EU, Dursun A, Beken S, Dilli D, Zenciroğlu A, Okumuş $\mathrm{N}$. Efficacy of single dose oral paracetamol in reducing pain during examination for retinopathy of prematurity: a blinded randomized controlled trial. Indian J Pediatr 2016;83:22-6.

5. Seifi F, Peirovifar A, Mostafa Gharehbaghi M. Comparing the efficacy of oral sucrose and acetaminophen in pain relief for ophthalmologic screening of retinopathy of prematurity. Am J Med Sci Med 2013;1:24-7.

6. Hansen A. Innovative approaches to assessment of pain in the NICU. https://clinicaltrials.gov/ct2/show/NCT03057782. Accessed 5 Feb 2019

7. Berde CB, Walco GA, Krane EJ, Anand KJS, Aranda JV, Craig $\mathrm{KD}$, et al. Pediatric analgesic clinical trial designs, measures, and extrapolation: report of an FDA scientific workshop. Pediatrics 2012;129:354-64.

8. Anand KJS, Aranda JV, Berde CB, Buckman S, Capparelli EV, Carlo W, et al. Summary proceedings from the neonatal paincontrol group. Pediatrics 2006;117(3 Pt 2):S9-22.

9. Shaddy RE, Denne SC, Research TC, on D and C on P. Guidelines for the ethical conduct of studies to evaluate drugs in pediatric populations. Pediatrics 2010;125:850-60. 